\title{
UM APLICATIVO COMPUTACIONAL PARA O ENSINO DE INTEGRAIS
} DUPLAS

\author{
Eleni Bisognin - eleni@unifra.br - UNIFRA \\ Leandro Ribeiro Fontoura - leandro.fontoura@unifra.br - UNIFRA
}

Resumo: Este trabalho buscou investigar as contribuições da utilização de um aplicativo computacional, programado com o suporte do software Maple, para o ensino e aprendizagem do conteúdo de integrais duplas. A pesquisa foi realizada com alunos do curso de Licenciatura em Matemática do Centro Universitário Franciscano de Santa Maria - RS. O referencial teórico para análise dos resultados baseou-se na teoria de Tall e Vinner (1981), sobre Conceito Imagem e Conceito Definição. A partir da aplicação de um teste diagnóstico, elaborou-se uma sequência didática para construir o conceito de integral dupla utilizando como recurso o aplicativo computacional nas aplicações referentes ao cálculo de volume de sólidos geométricos. Os dados foram obtidos por meio da observação, dos registros feitos pelo professor em seu diário de campo e dos documentos produzidos pelos alunos. Os resultados demonstraram que, a utilização do aplicativo computacional auxiliou na visualização das imagens e na elaboração de simulações para a formação do conceito de integral dupla e auxiliou os alunos no cálculo do volume dos sólidos geométricos.

Palavras-chave: Aplicativo Computacional; Conceito imagem e Conceito definição; Integral Dupla.

\section{A COMPUTATIONAL APPLICATION FOR THE TEACHING OF DOUBLE INTEGRALS}

\begin{abstract}
This work aimed to investigate the contributions of the use of a computational application, programmed with Maple software help, for the teaching and learning processes of the double integrals content. The research was done with students from the Mathematics Course from at Centro Universitário Franciscano in Santa Maria RS. The theoretical referential for the results analysis was based on the theory of Tall and Vinner (1981), about the Image and Definition Concepts. From the application of this diagnostic test, a didactical sequence was built to construct the concept of double integral using the computational application as a resource in the applications referring to the volume calculation of geometric solids. The data was obtained through observation, registration done by the teacher in his field diary and the documents produced by the students. The results show that, the use of the computational application helped in the images visualization and in the elaboration of simulations for the creation of the double integral concept and helped students in the volume calculation of the geometric solids.
\end{abstract}

Keywords: Computer application; Concept Image and Concept Definition; Double Integral.

\section{Introdução}

De acordo com resultados de pesquisas como (Igliori, 2009), (Dalla Porta, 2014) e (Nasser, 2006), as disciplinas de Cálculo têm altos índices de rendimento 
insatisfatório, evasão e reprovação. Segundo os autores, uma das principais razões para isso é a pouca motivação dos alunos para o estudo desses tópicos, fruto de aulas expositivas e pouco motivadoras, pouco uso de recursos computacionais que auxiliem na visualização, entre vários outros fatores.

Segundo (Borba e Penteado, 2001), a informática não melhora nem piora o ensino, ela transforma o ensino e transforma a aprendizagem, transformando a forma como as pessoas produzem conhecimento. Dessa forma, a informática transforma os modos de ensinar e aprender, bem como a forma de as pessoas produzirem conhecimento. Nesse sentido, os mesmos autores salientam que o aspecto visual é importante em conteúdos matemáticos, como interpretação de gráficos de funções ou geometria, pois favorece experimentações e aproxima os alunos da Matemática.

O caráter estático das representações matemáticas, características da metodologia tradicional de ensino, que se baseia na memorização de conteúdos e definições, na repetição de processos predeterminados de fazer Matemática, como algoritmos, e no procedimento repetitivo como modo de conhecer e fazer, não têm significado para o aluno que, atualmente, vive num mundo rodeado de transformações e informações.

A utilização do Software Maple viabiliza uma melhor aprendizagem, por auxiliar a construção de conceitos antes vistos de uma forma meramente expositiva. A visualização, o movimento e as simulações fazem com que o aluno compreenda o conteúdo de uma forma interativa e melhor elaborada devido a todas as capacidades e potencialidades que o software proporciona.

Por acreditar no potencial didático do software e na tentativa de buscar novas formas de produzir conhecimento matemático utilizando-se metodologias adequadas, colocou-se a seguinte questão de pesquisa: que contribuições podem oferecer para o ensino e aprendizagem dos conceitos e propriedades da integral dupla a utilização da metodologia da Engenharia Didática com o apoio computacional do software Maple?

Além dos recursos computacionais, a utilização de metodologias adequadas contribui para a motivação e compreensão dos conceitos pelos estudantes. Dessa maneira, elaborou-se uma sequência didática, apoiada nos pressupostos teóricos da Engenharia Didática, para o ensino e aprendizagem de conceitos e propriedades da integral dupla, utilizando o aplicativo desenvolvido no software Maple como ferramenta de apoio para os cálculos de volumes e para a visualização dos sólidos.

Para análise dos dados foi utilizada a teoria de Tall e Vinner sobre Conceito Imagem e Conceito Definição. Dos resultados obtidos pode-se inferir que a utilização do aplicativo computacional foi fundamental para a construção e compreensão dos conceitos sobre integral dupla.

\section{Engenharia Didática}

A Engenharia Didática de acordo com (Artigue, 1996), tem sua origem na Didática da Matemática Francesa, no início dos anos 1980 e é uma metodologia de trabalho didático análoga ao trabalho do engenheiro. De acordo com (Douady, 1993) citada por (Machado, 2002) o termo Engenharia Didática é entendido como:

[...] uma sequência de aulas concebidas, organizadas e articuladas no tempo, de forma coerente, por um professor engenheiro para realizar um projeto de aprendizagem para uma certa população de alunos. No decurso das trocas entre professor e aluno, o projeto evolui sob as reações dos alunos e em função das escolhas e decisões do professor. (Machado, 2002, p.198). 
Como metodologia de pesquisa, se caracteriza pela aplicação de uma sequência de aulas planejadas com a finalidade de obter informações que permitam interpretar processos de ensino e aprendizagem da Matemática, elucidando o fato investigado.

No que tange uma produção para o ensino a aprendizagem, é vista como um processo de adaptação que depende das características das situações em que o ensino se desenvolve. As produções para o ensino são derivadas de resultados de pesquisas e também a Engenharia Didática é uma metodologia de pesquisa baseada em experiências de sala de aula. Segundo (Carneiro, 2005), a prática de ensino é articulada com prática de investigação. A metodologia da Engenharia Didática pode ser vista como referencial para o desenvolvimento de produtos para o ensino, gerados na junção do conhecimento prático com o conhecimento teórico.

A estrutura metodológica para uma Engenharia Didática, segundo (Artigue, 1996), compreende quatro etapas, quais sejam: a) análise preliminar, que consiste no levantamento das concepções envolvidas e a busca de referencial teórico que fundamentam a pesquisa; b) análise a priori, que consiste no processo de construção e elaboração de material e atividades; c) experimentação, que compreende a aplicação da sequência didática; d) análise a posteriori e validação da experiência. Corresponde à análise do conjunto dos dados obtidos na fase da experimentação e das observações realizadas durante a aplicação da sequência.

A primeira etapa compreende os aspectos epistemológicos do conteúdo a ser trabalhado. Nessa fase, podemos observar como os alunos se apropriaram do conteúdo trabalhado e quais suas dificuldades. Dessa forma, foi aplicado um teste diagnóstico buscando explorar o conhecimento que eles possuíam sobre o cálculo de área e de volume. Também, incluiu-se a análise de livros didáticos utilizados na disciplina de Cálculo Diferencial e Integral.

De acordo com (Artigue, 1996), a finalidade dessa etapa é determinar quais relações, de fato, permitem controlar os comportamentos dos estudantes e seus significados.

A segunda etapa, concepção e análise a priori, aborda o processo de construção e elaboração das atividades que serão experimentadas, objetivando os alunos e o objetivo proposto. Trata-se do processo de construção e elaboração do material e das atividades propostas.

A terceira etapa corresponde à experimentação, ou seja, a intervenção didática junto aos alunos. No decorrer dessa etapa, é de fundamental importância que o professor/pesquisador seja cuidadoso no desenvolvimento da aula, considerando todas as informações que irão subsidiar a pesquisa. Para o registro dessas informações, podese fazer uso dos seguintes recursos: gravações, filmagens, diário de campo, entre outros. Tal cuidado é importante para que o relatório seja fidedigno ao transcorrido nas aulas. A próxima etapa é a de análise a posteriori, a qual se apoia no conjunto de dados recolhidos durante a experimentação. Trata-se da compreensão dos resultados obtidos e seu objetivo é oferecer um retorno para o desenvolvimento de uma nova análise $a$ priori, para uma nova experimentação, concebendo o desenvolvimento das atividades como uma atualização dos processos em questão.

\subsection{Conceito Imagem e Conceito Definição}

Entre as teorias cognitivas relativas à construção dos conceitos matemáticos, tem-se a teoria desenvolvida por (Tall e Vinner, 1981), referente ao Conceito Imagem e Conceito Definição. Essa teoria preconiza que um determinado conceito matemático 
não deve ser ensinado ao estudante tendo como única referência pedagógica sua definição formal. Para os autores, uma definição formal será compreendida de maneira satisfatória pelo estudante, quando existe uma familiarização anterior com o conceito em questão, desenvolvida com base em impressões e experiências diversas.

Segundo (Tall e Vinner, 1981, p. 152), o termo Conceito Imagem é utilizado

[...] para descrever a estrutura cognitiva total associada ao conceito, que inclui todas as figuras mentais, processos e propriedades associados. Ela é construída ao longo dos anos, através de experiências de todos os tipos, mudando enquanto o indivíduo encontra novos estímulos e amadurece (Tall; Vinner, 1981, p. 152).

De acordo com (Tall, 1988) as noções matemáticas não são apenas utilizadas de acordo com suas definições formais, mas também por meio de representações mentais que podem variar de pessoa para pessoa. De tal modo, o conceito imagem é continuamente construído pelo sujeito por meio de todo tipo de experiência vivenciada, podendo ser alterado ao longo do desenvolvimento cognitivo do sujeito.

Para (Giraldo, 2004), o conceito imagem de um indivíduo não é uma estrutura estática e, por isso, ela passa por transformações conforme o desenvolvimento cognitivo do sujeito, podendo incluir, excluir ou modificar atributos, enriquecendo-os no decorrer de suas experiências cognitivas.

Para (Tall e Vinner, 1981), o Conceito Definição é um problema significativamente diferente, determinado por:

[...] Uma reconstrução pessoal feita pelo estudante. É então o tipo de palavras que o estudante usa para sua própria explanação de seu conceito imagem. Se os conceitos definição lhe são dados ou construídos por si mesmo, podem variar de tempo em tempo. Dessa maneira, um conceito definição pessoal pode ser diferente de um conceito definição formal, este último sendo um conceito definição que é aceito pela comunidade matemática (Tall; Vinner, 1981, p.153).

Ainda segundo (Vinner, 1991, p.69): "Adquirir um conceito significa formar uma imagem de conceito". Isto é, para a formação de determinado conceito, é preciso criar distintas imagens conceituais; após a criação de imagens sobre determinados conceitos é que o sujeito tem condições de descrevê-la com o uso de palavras conduzindo para a definição de conceito. Porém, o autor afirma que conceitos cotidianos, como casa, carro, cachorro, entre outros, podem ser obtidos sem envolver uma definição e, mesmo assim, o conceito imagem é bastante claro. Por outro lado, alguns conceitos podem ser introduzidos por meio de uma definição, auxiliando a formação de uma imagem. Por exemplo, quando é dito para uma criança que floresta é uma região com uma grande quantidade de árvores, a criança pode formar um conceito imagem a partir dessa definição. No entanto, quando o conceito imagem está formado, a definição pode permanecer inativa ou, até mesmo, esquecida.

Em relação aos contextos técnicos, (Vinner, 1991) considera que definições podem exercer papéis extremamente importantes, não somente porque elas ajudam a formar um conceito imagem, mas porque, frequentemente, têm um papel decisivo na tarefa cognitiva, ou seja, na organização formal e conexão das ideias, essenciais para a compreensão expressiva dos conteúdos.

Dessa forma, a apresentação de um conceito deve ser antecedida por uma abordagem pedagógica que possibilite a formação do conceito imagem rico e 
consistente relacionado ao conceito. Assim, a compreensão e a assimilação da definição formal aconteceriam de forma mais significativa para o aluno.

\subsection{Aplicativo desenvolvido no Software Maple para Integrais Duplas}

De acordo com (Moren; Santos, 2011) o uso de um recurso computacional deve favorecer a construção do conhecimento e valorizar a inovação e a descoberta. Nesta perspectiva, o professor pode explorar novas possibilidades didáticas e metodológicas, incluindo momentos de experiências laboratoriais que permitam a migração da cadeia formal do ensino tradicional do Cálculo Diferencial e Integral - representada pela sequência "definição $\rightarrow$ teorema $\rightarrow$ demonstração $\rightarrow$ corolário (aplicações)", para a cadeia exploratória caracterizada pelos passos: "exploração $\rightarrow$ conjectura $\rightarrow$ tentativa de demonstração $\rightarrow$ conclusão e aplicação", transformando o aluno de paciente em agente do processo educativo. (Santos et al., 2004).

O software Maple disponibiliza ferramentas de Clickable Math (matemática clicável), com menus sensíveis ao contexto, permitindo ao professor gerar aplicativos interativos, com a exigência de prévios conhecimentos computacionais.

Com o objetivo de utilizar, neste trabalho, uma ferramenta computacional que facilite o ensino e a aprendizagem do conteúdo de integrais duplas, optou-se por desenvolver o aplicativo de maneira que o aluno consiga visualizar regiões do plano, faça a construção de sólido e, por último, particione o sólido em paralelepípedos, para estudar o volume do sólido por meio de somas aproximadas do volume dos paralelepípedos.

A Figura 1 representa a parte do aplicativo em que devem ser inseridas as informações para a representação da região no plano.

Figura 1 - Aplicativo desenvolvido no software Maple para representar a região do plano

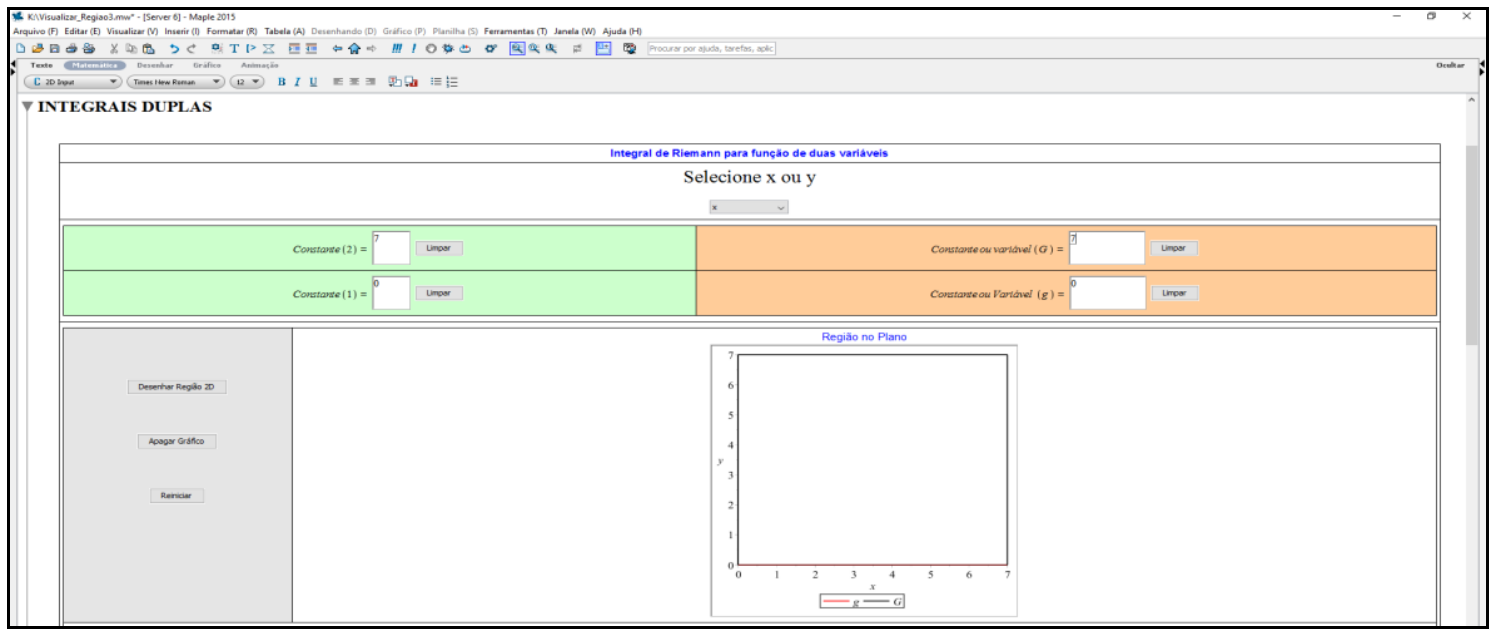

Fonte: Autor.

Após a construção da região do plano, insere-se a função a ser integrada para construção, visualização e manipulação do sólido que se deseja calcular o volume. Por exemplo, ao escrever a função $\mathrm{f}(\mathrm{x}, \mathrm{y})=\mathrm{x}+\mathrm{y}$ obtém-se o sólido mostrado na figura 2 , a seguir. 
Figura 2 - Sólido gerado no aplicativo

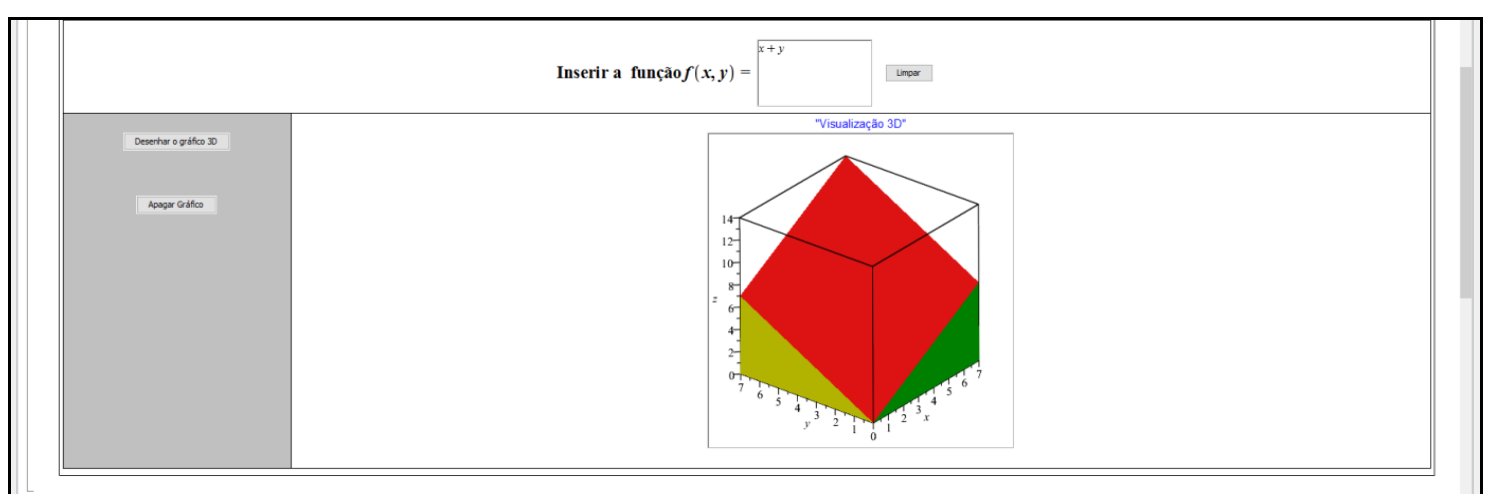

Fonte: Autor.

Por último, o sólido é particionado em paralelepípedos, e pode-se calcular a aproximação do volume do sólido por somas superiores ou por somas inferiores.

Figura 3 - Aplicativo do software Maple para aproximar o volume de uma integral dupla

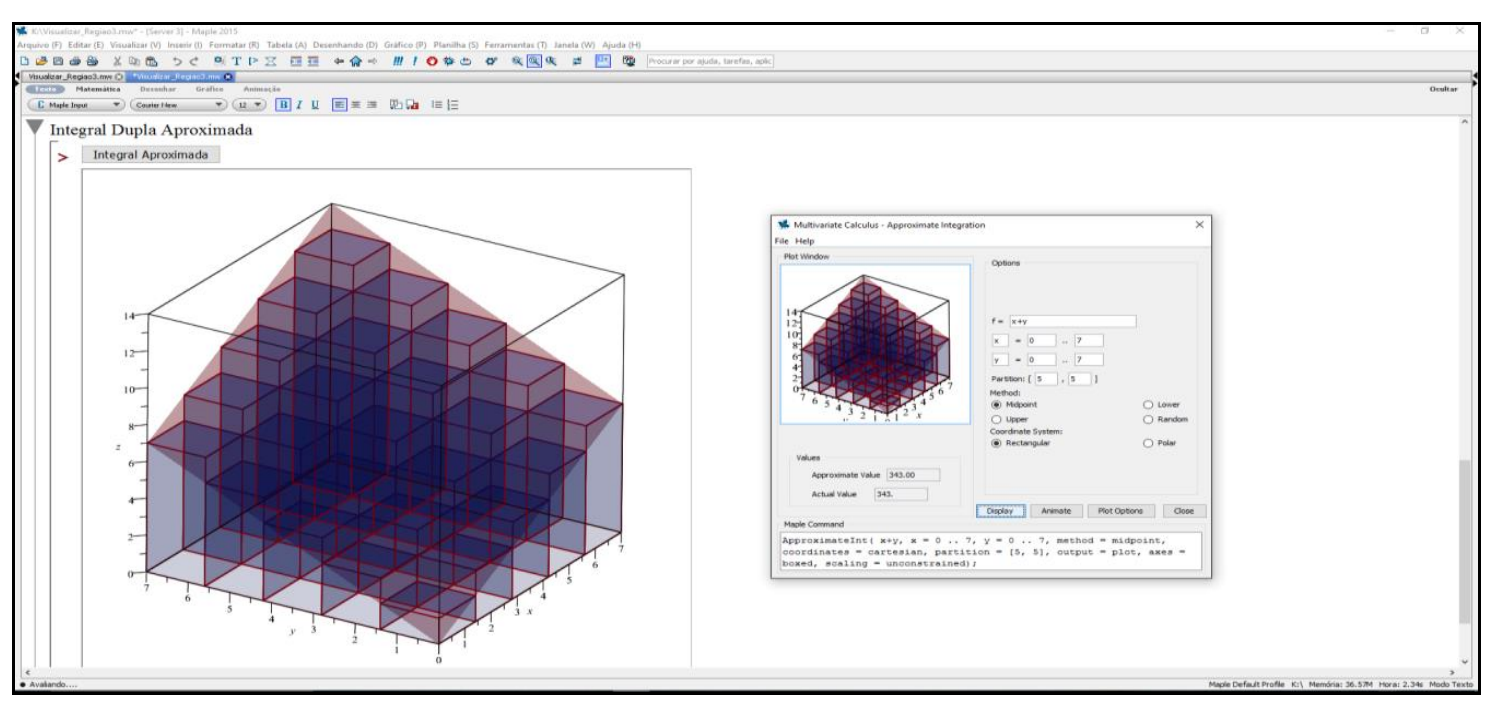

Fonte: Autor.

\section{METODOLOGIA}

A fundamentação teórica para a construção da sequência didática se deu por meio da análise de dois livros didáticos e de um teste diagnóstico realizado com os alunos. Os livros analisados são de autoria de (Anton, 2014) e (Stewart, 2013).

A pesquisa foi desenvolvida com cinco alunos do quinto semestre do Curso de Licenciatura em Matemática, matriculados na disciplina de Cálculo IV, de uma universidade particular de Santa Maria, RS. Os alunos trabalharam individualmente no laboratório de informática do curso. A utilização do laboratório de informática foi necessária para executar, no software Maple, o aplicativo desenvolvido pelo professor pesquisador como ferramenta de apoio para a visualização das regiões no plano e dos sólidos. Ao final de cada atividade, foi reservado um tempo para discussão e análise, com os alunos, dos exercícios propostos.

Com os resultados obtidos da análise do teste diagnóstico, o qual teve como objetivo a identificação das possíveis dificuldades em relação aos conceitos de área e 
volume, elaborou-se uma sequência didática, composta de quatro atividades para construir o conceito de integral dupla, apoiada no aplicativo computacional desenvolvido no software Maple.

\section{RESULTADOS E DISCUSSÕES}

A primeira atividade da sequência didática objetivou conceituar a integral dupla, de maneira que os alunos pudessem calcular o volume de um sólido por meio da soma dos volumes dos paralelepípedos. Para isso, iniciou-se com o cálculo do volume de um cubo por meio da geometria espacial; posteriormente, esse cubo foi particionado em paralelepípedos, para que os alunos percebessem que a soma do volume dos paralelepípedos resultaria no volume do cubo. Em seguida, considerou-se um sólido limitado superiormente por um plano não paralelo à base para se calcular o volume aproximado, dividindo a área da base em pequenos sub-retângulos e calculando o volume por meio de paralelepípedos retângulos, por excesso e por falta. Em vista disso, pretendia-se que os alunos compreendessem o processo da construção das somas de Riemann, para, a seguir, conseguirem formar o conceito de integral dupla.

Tendo em vista as dificuldades apresentadas durante a aplicação da atividade, o professor pesquisador utilizou o aplicativo disponível do software Maple, para realizar uma animação computacional revelando o conceito proposto de forma mais lúdica.

A animação foi apresentada e discutida no encerramento da primeira atividade. Essa animação mostrou a construção do sólido e o cálculo aproximado do volume, em que foi utilizado um número cada vez maior de paralelepípedos para particionar o sólido proposto. Essa animação é mostrada nas imagens da figura 4,a seguir.

Figura 4 - Aproximação do volume do sólido utilizando o software Maple

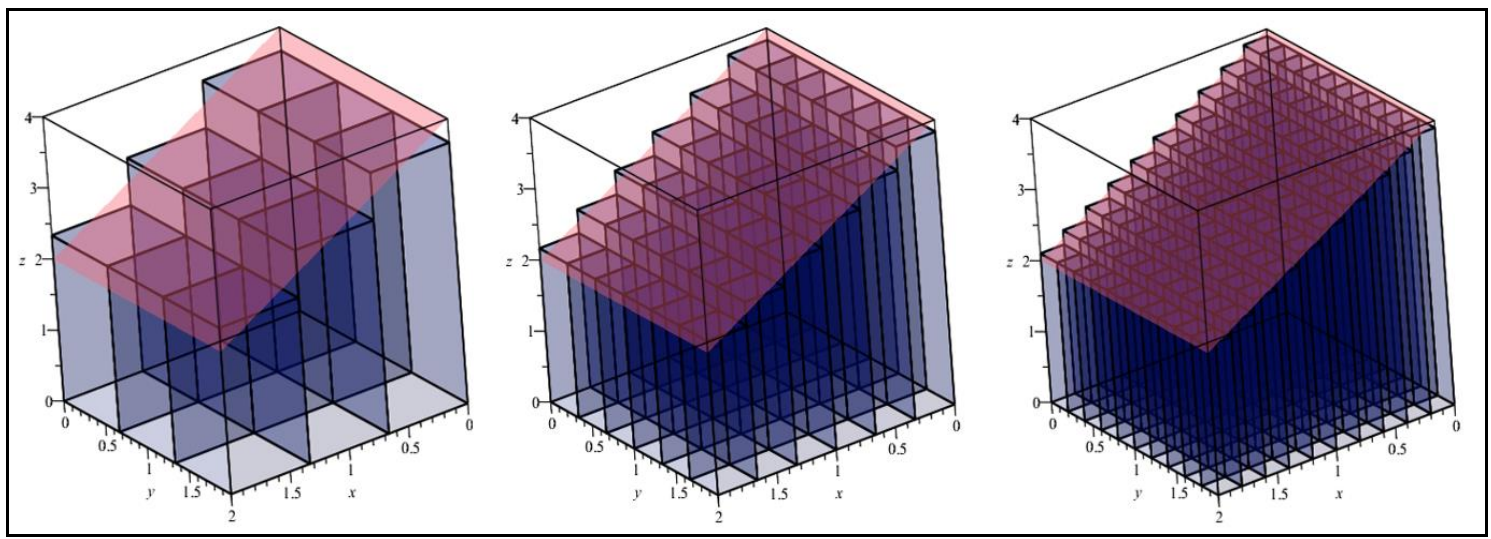

Fonte: Autor

Constatou-se que a apresentação da animação foi positiva, pois os alunos puderam esclarecer as inúmeras dúvidas que foram mostradas nas análises das atividades aplicadas. Eles interagiram durante a apresentação e conseguiram, de forma satisfatória, construir o conceito da integral dupla com o auxílio do professor pesquisador.

A segunda atividade teve como propósito mostrar e analisar o processo de aproximação do volume de um sólido com a base retangular, por meio da soma do volume dos paralelepípedos, e, posteriormente, confrontar o resultado obtido por meio do cálculo da integral dupla. Para auxiliar o cálculo das somas de Riemann foi utilizada 
a planilha eletrônica Microsoft Excel, e do aplicativo desenvolvido pelo professor pesquisador, como ferramenta de apoio para visualização.

A terceira e quarta atividades tinham como objetivo a construção e consolidação do conceito da integral dupla, para o cálculo do volume de sólidos em regiões com base não retangular. Em especial, a construção de gráficos para a determinação dos limites de integração da região do plano e a visualização do sólido para posterior cálculo do volume utilizando uma integral iterada. Nessa etapa a utilização do aplicativo foi fundamental para compreensão do processo de construção do conceito.

\section{CONCLUSÃO}

O desenvolvimento desta pesquisa objetivou analisar as contribuições da utilização de um aplicativo computacional, programado com o suporte do software Maple, para o ensino e aprendizagem do conteúdo de integrais duplas.

Entende-se que algumas representações gráficas são difíceis de serem visualizadas utilizando métodos como papel e lápis, lousa e giz, ou, ainda, que os livros didáticos fornecem representações em suas definições e exemplos, porém limitadas, não proporcionando a manipulação das informações para uma nova investigação. Nesse sentido, a utilização do aplicativo computacional viabilizou uma melhor aprendizagem pois auxiliou na construção dos conceitos antes vistos de uma forma meramente expositiva. De acordo com (Morelatti, 2001), nas disciplinas de Cálculo Diferencial e Integral, o computador é um simulador de conjecturas e uma ferramenta de visualização de conceitos para melhor compreensão do ensino e aprendizagem.

Durante o desenvolvimento das atividades quando se propôs conceituar a integral dupla e aproximar o volume por meio da soma dos volumes dos paralelepípedos por excesso e por falta, identificaram-se muitas dificuldades no processo de construção do conceito. Essas dificuldades de aprendizagem dos alunos acreditam-se que foram causadas por muitos fatores, como por exemplo, imprecisão ao expressarem-se matematicamente, não domínio de conteúdos básicos, como partição, cálculo de áreas de figuras planas e cálculo do volume. A análise dos resultados dessas atividades mostrou fragilidades, mas também mostrou a eficácia da utilização do aplicativo computacional, pois a visualização e as simulações propiciadas pelo aplicativo auxiliaram os alunos na identificação da região de integração, na aproximação do volume pelas somas de Riemann e na determinação dos limites de integração. O uso da planilha eletrônica Excel também tornou mais simples o cálculo das somas de Riemann.

Pode-se concluir que a utilização do aplicativo computacional facilitou a formação de Conceito Imagem e Conceito Definição, sobre o conteúdo de integrais duplas e auxiliou os alunos no cálculo do volume de sólidos geométricos.

\section{REFERÊNCIAS}

ANTON, H. Cálculo, Volume 2. 10ª . ed. Porto Alegre: Bookmann, 2014.

ARTIGUE, M. Engenharia Didática. In:BRUN, J. Didáctica das Matemáticas. Lisboa: Instituto Piaget.Horizontes Pedagógicos, 1996, p.193-217.

BORBA, M. C.; PENTEADO, M. G. Informática e Educação Matemática. Belo

Horizonte: Autêntica, 2001. Coleção Tendências em Educação Matemática. 
CARNEIRO, V. C. G. Engenharia didática: um referencial para ação investigativa e para formação de professores de Matemática. Zetetike, Campinas, v. 13, n. 23, p. 85-118, 2005.

DALLA PORTA, L. Contribuições da engenharia didática para o ensino e aprendizagem de funções de várias variáveis reais. Dissertação (Mestrado Profissionalizante em Ensino de Física e de Matemática) - Centro Universitário Franciscano de Santa Maria, 2014.

GIRALDO, V. Descrições e conflitos computacionais: o caso da derivada. Tese (Doutorado em Ciências) - COPPE. Rio de Janeiro: UFRJ, 2004.

IGLIORI, S. B. C. Considerações sobre o ensino do cálculo e um estudo sobre os números reais. In FROTA, M. C. R; NASSER, L. (Orgs.) Educação Matemática no Ensino Superior: pesquisas e debates. Recife: SBEM, 11 - 26, 2009.

MACHADO, S. D. A. et al. Engenharia didática in: Educação Matemática: uma introdução. São Paulo: EDUC, 2002, p. 197-208.

MORELATTI, M.R.M. Criando um ambiente construcionista de aprendizagem em Cálculo Diferencial e Integral I. Tese (Doutorado em Educação) - Programa: Supervisão e Currículo, PUCSP. São Paulo, 2001

MOREN, E. B. S., SANTOS, A. R. Uma reflexão sobre ações de formação de professores no Brasil. Revista Iberoamericana de Educación(online), v. 55, p. 11, 2011.

NASSER, L.: Aprimorando o desempenho de alunos de Cálculo no traçado de gráficos. Atas do III SIPEM (em CD). SBEM, 2006.

SANTOS, A. R., KUBRUSLY, R. \& BIANCHINI, W. Mathlets: Applets Java para o ensino de Matemática. In C. A. Moura, H. Noronha, J. A. Fossa, L.M. Carvalho e V. Giraldo (Eds), História e Tecnologia no Ensino da Matemática. Volume 2. (pp. 323336). Rio de Janeiro, Brasil: Editora Ciência Moderna. 2004.

STEWART, J. Cálculo, Volume 2. 7ª ed. São Paulo: Cengage Learning

TALL, D. O. Concept image and concept definition. In: David Tall Home Page. Disponível em: http://www.warwick.ac.uk/staff/David.Tall/themes/conceptimage.html, 2003. Acesso em: 12/02/2016

TALL, D. O. Concept image and Concept definition. In: Senior Secondary Mathematics Education, p. 37 - 41, 1988.

TALL, D. O. VINNER, S. Concept image and concept definition in mathematics, with special reference to limits and continity. Educational Studies in Mathematics, n. 12, p. 151-169, 1981. 
VINNER, S. The role of definitions in the teaching and learning of mathematics, In:

TALL D. O. (Ed). Advanced Mathematical Thinking. Dordrecht: Kluwer Academic Publishers, p.65-81,1991. 